\title{
HOPF MANIFOLDS AND SPECTRAL GEOMETRY
}

BY

\author{
KAZUMI TSUKADA
}

\begin{abstract}
We characterize Hopf manifolds in the class of Hermitian manifolds by the spectra of the real Laplacians and the complex Laplacians.
\end{abstract}

1. Introduction. Let $M$ be an $n$-dimensional compact connected Hermitian manifold. We define two differential operators on $M$ i.e., the real Laplacian $\Delta_{r}$ acting on $r$-forms and the complex Laplacian $\square_{p, q}$ acting on forms of type ( $\left.p, q\right)$ (cf. §3). We denote by $\operatorname{Spec}\left(M, \Delta_{r}\right)\left(\operatorname{resp} . \operatorname{Spec}\left(M, \square_{p, q}\right)\right)$ the set of eigenvalues of $\Delta_{r}$ (resp. $\square_{p, q}$ ) enumerated with multiplicity. It is an interesting problem whether the spectrum of the Laplacian determines the geometry of the manifold. In this note we study the spectral geometry of Hermitian manifolds known as the Hopf manifolds which are not Kaehler.

Definition of The Hopf Manifolds $M_{\alpha}$ (CF. E. Bedford and T. SUwa [1]). Let $W$ be the $n$-dimensional complex vector space $\mathbf{C}^{n}=\left\{z \mid z=\left(z_{1}, \ldots, z_{n}\right)\right\}$ minus the origin; $W=\mathbf{C}^{n}-\{0\}$, and let $\alpha$ be a complex number with $0<|\alpha|<1$. The Hopf manifold $M_{\alpha}$ is defined as $M_{\alpha}=W / G_{\alpha}$, where $G_{\alpha}$ is the group generated by the analytic automorphism $z \rightarrow \alpha z, z \in W . M_{\alpha}$ is well known as an example of a compact complex manifold which does not admit Kaehler metrics. On the manifold $M_{\alpha}$, there is a natural Hermitian metric given by

$$
g=\|z\|^{-2} \sum_{j=1}^{n} d z_{j} d \bar{z}_{j}, \quad\|z\|^{2}=\sum_{k=1}^{n} z_{k} \bar{z}_{k} .
$$

From now on we think of $M_{\alpha}$ as a Hermitian manifold with this metric.

E. Bedford and T. Suwa [1] described explicitly the eigenvalues of $\Delta_{0}$ and $\square_{0,0}$ on the Hopf manifolds $M_{\alpha}$. They also obtain some isospectral results in the class of the Hopf manifolds.

In this paper, we show

THEOREM. Let $M_{\alpha}$ be an $n$-dimensional $(n \geqslant 3)$ Hopf manifold with $-\frac{1}{2 \pi} \operatorname{Re} \log \alpha>$ $1 / 2 \sqrt{2 n+1}$. If a compact connected Hermitian manifold $M$ satisfies $\operatorname{Spec}\left(M, \Delta_{r}\right)=$ $\operatorname{Spec}\left(M_{\alpha}, \Delta_{r}\right)$ and $\operatorname{Spec}\left(M, \square_{p, q}\right)=\operatorname{Spec}\left(M_{\alpha}, \square_{p, q}\right)$ for $r=0,1$ and $(p, q)=(0,0)$, $(0,1),(1,0)$, then $M$ is biholomorphically isometric to $M_{\alpha}$.

The author wishes to thank Professor K. Ogiue for his helpful advice.

Received by the editors December 19, 1980.

1980 Mathematics Subject Classification. Primary 53C55, 58G25.

Key words and phrases. Hopf manifolds, Hermitian metric, 1.c.K. manifolds, Laplacian, eigenvalues, isospectral problem. 
2. Some classes of Hermitian manifolds. We define two classes of Hermitian manifolds, i.e., semi-Kaehler manifolds and 1.c.K. manifolds. Let $(M, g, J)$ be a Hermitian manifold with Hermitian metric $g$ and complex structure $J$ and $\Omega$ be the 2-form defined by $\Omega(X, Y)=g(J X, Y)$, which is called the fundamental 2-form of $(M, g, J)$.

DEFINITION 2.1. An $n$-dimensional Hermitian manifold $(M, g, J)$ is called semiKaehler if it satisfies $\delta \Omega=0$ or, equivalently, $d \Omega^{n-1}=0$, where $\delta$ denotes the codifferential operator with respect to the metric $g$.

For examples of semi-Kaehler manifolds, we refer to P. Gauduchon [4] and A. Gray [7], etc.

Definition 2.2. A Hermitian manifold $(M, g, J)$ is called locally conformal Kaehler (l.c.K.) if its metric is conformally related to a Kaehler metric in some neighborhood of every point of $M$.

An interesting example of l.c.K. manifolds is given by the Hopf manifolds $M_{\alpha}$. A characterization of the l.c.K. manifolds is

$$
d \Omega=2 \omega \wedge \Omega
$$

where $\omega$ is a well-defined closed 1-form called the Lee form (cf. I. Vaisman [14]). For l.c.K. manifolds, we refer to I. Vaisman $[14,15]$ and T. Kashiwada [8], etc.

Here we recall the Hermitian connection on Hermitian manifolds.

Proposition 2.3 (KobAyAShi AND Nomizu [9]). On a Hermitian manifold $(M, g, J)$, there exists a unique linear connection $\nabla$ which satisfies the following properties.

(1) $g$ and $J$ are parallel, i.e., $\nabla g=0$ and $\nabla J=0$.

(2) The torsion tensor $S$ is pure in the following sense: $S(J X, Y)=S(X, J Y)$ for all vector fields $X, Y$ on $M$.

The linear connection defined in Proposition 2.3 is called the Hermitian connection of the Hermitian manifold $M$.

Let $\left(z_{1}, \ldots, z_{n}\right)$ be a local coordinate system for $M$ and set

$$
g_{\alpha \bar{\beta}}=g\left(\partial / \partial z_{\alpha}, \partial / \partial \bar{z}_{\beta}\right) \text {. }
$$

We denote by $\left(g^{\bar{\beta} \alpha}\right)$ the inverse matrix of $\left(g_{\alpha \bar{\beta}}\right)$. Then we have the following formulas for covariant differentiation:

$$
\nabla_{\partial / \partial z_{\alpha}}\left(\frac{\partial}{\partial z_{\beta}}\right)=\sum_{\gamma} \Gamma_{\alpha \beta}^{\gamma} \frac{\partial}{\partial z_{\gamma}}, \quad \nabla_{\partial / \partial \bar{z}_{\alpha}} \frac{\partial}{\partial z_{\beta}}=0
$$

where

$$
\Gamma_{\alpha \beta}^{\gamma}=\sum_{\lambda} g^{\bar{\lambda} \gamma} \frac{\partial g_{\beta \bar{\lambda}}}{\partial z_{\alpha}}
$$

The torsion tensor $S$ is given by

$$
S_{\alpha \beta}^{\gamma}=\Gamma_{\alpha \beta}^{\gamma}-\Gamma_{\beta \alpha}^{\gamma}
$$


We define a new tensor field $T$ of type $(1,2)$ by

$$
\begin{aligned}
& T_{\beta \gamma}^{\alpha}=S_{\beta \gamma}^{\alpha}+\left(\sum_{\mu} S_{\mu \beta}^{\mu} \delta_{\gamma}^{\alpha}-\sum_{\lambda} S_{\lambda \gamma}^{\lambda} \delta_{\beta}^{\alpha}\right) /(n-1), \\
& T_{\bar{\beta} \bar{\gamma}}^{\bar{\alpha}}=S_{\bar{\beta} \bar{\gamma}}^{\bar{\alpha}}+\left(\sum_{\mu} S_{\mu \beta}^{\bar{\mu}-} \delta_{\gamma}^{\alpha}-\sum_{\lambda} S_{\lambda \bar{\gamma}}^{\bar{\alpha}} \delta_{\beta}^{\alpha}\right) /(n-1)
\end{aligned}
$$

and other $T_{B C}^{A}=0$.

We define a 1 -form

$$
\theta=\sum_{\alpha} \theta_{\alpha} d z_{\alpha}+\sum_{\alpha} \theta_{\bar{\alpha}} d \bar{z}_{\alpha}
$$

by

$$
\theta_{\alpha}=\sum_{\lambda} S_{\lambda \alpha}^{\lambda} \text { and } \quad \theta_{\bar{\alpha}}=\sum_{\mu} S_{\mu \bar{\alpha}}^{\bar{\mu}}
$$

Proposition 2.4. Let $M$ be an n-dimensional Hermitian manifold and $S$ the torsion tensor of the associated Hermitian connection. Then:

(1) $S$ vanishes if and only if $M$ is Kaehler.

(2) The 1-form $\theta$ vanishes if and only if $M$ is semi-Kaehler.

(3) When $n \geqslant 3$, the tensor field T vanishes if and only if $M$ is l.c.K..

Proof. (2) After straightforward calculations we obtain $\theta(X)=\delta \Omega(J X)$.

(3) $T \equiv 0$ implies that the 1 -form $\theta$ is closed. The torsion tensor $S$ and the fundamental 2-form $\Omega$ are related by

$$
(d \Omega)_{\alpha \beta \bar{\gamma}}=\frac{1}{3} \sqrt{-1} g_{\lambda \bar{\gamma}} S_{\alpha \beta}^{\lambda}, \text { etc. }
$$

Therefore if we set $\omega=-\theta / 2(n-1), T \equiv 0$ is equivalent to the formula $(*)$.

REMARK (i). On a 2-dimensional Hermitian manifold $M, T$ vanishes identically. In this case, in order that a 2-dimensional Hermitian manifold be 1.c.K., it is necessary and sufficient that the 1 -form $\theta$ be closed.

REMARK (ii). It follows by Proposition 2.4 that if a Hermitian manifold $M$ is semi-Kaehler and l.c.K., then $M$ is Kaehler (cf. Vaisman [16]).

We denote by $\tau$ and $\tilde{\tau}$ the scalar curvatures with respect to the Riemannian connection and the Hermitian connection, respectively. We set $S_{\beta \gamma, \lambda}^{\alpha}=\nabla_{\lambda} S_{\beta \gamma}^{\alpha}$, where $\nabla$ is the covariant differentiation of the Hermitian connection. Then we have the following.

PROPOSITION 2.5.

$$
\tau=\tilde{\tau}-\frac{1}{2} S_{\beta \gamma}^{\alpha} S_{\bar{\beta} \bar{\gamma}}^{\bar{\alpha}}-2 S_{\lambda \alpha}^{\lambda} S_{\mu \bar{\alpha}}^{\bar{\mu}}+2 S_{\alpha \beta, \bar{\beta}}^{\alpha} \text {, }
$$

where $S_{\beta \gamma}^{\alpha} S_{\bar{\beta} \bar{\gamma}}^{\bar{\alpha}}$, etc. stand for $g_{\alpha \bar{\lambda}} g^{\beta \bar{\mu}} g^{\gamma \bar{\kappa}} S_{\beta \gamma}^{\alpha} S_{\bar{\mu} \bar{\kappa}}^{\bar{\lambda}}$, etc.

(2) If $\operatorname{dim}_{\mathbf{C}} M \geqslant 3$, then

$$
S_{\beta \gamma}^{\alpha} S_{\bar{\beta} \bar{\gamma}}^{\bar{\alpha}}-(2 / n-1) S_{\lambda \alpha}^{\lambda} S_{\mu \bar{\alpha}}^{\bar{\mu}} \geqslant 0
$$


and the equality holds everywhere if and only if $M$ is l.c. $K$. Moreover, assume that $M$ is compact and denote by $d V_{g}$ the volume element with respect to the Riemannian metric g. Then

$$
\begin{gathered}
\int_{M} S_{\lambda \alpha}^{\lambda} S_{\mu \bar{\alpha}}^{\bar{\mu}} d V_{g}=\int_{M} S_{\alpha \beta, \bar{\beta}}^{\alpha} d V_{g}, \\
\int_{M} \tau d V_{g}=\int_{M} \tilde{\tau} d V_{g}-\frac{1}{2} \int_{M} S_{\beta \gamma}^{\alpha} S_{\bar{\beta} \bar{\gamma}}^{\bar{\alpha}} d V_{g}, \\
\int_{M} \tilde{\tau} d V_{g} \geqslant \int_{M} \tau d V_{g}
\end{gathered}
$$

with equality if and only if $M$ is Kaehler.

Proof. (1) is obtained by straightforward calculations.

(2) Computing the length of the tensor field $T$, we have this inequality.

(3) Calculating $\delta \theta$ and $\delta^{2} \Omega$, we have

$$
\delta \theta=2 S_{\lambda \alpha}^{\lambda} S_{\mu \bar{\alpha}}^{\bar{\mu}}-S_{\alpha \beta, \bar{\beta}}^{\alpha}-S_{\bar{\alpha} \beta, \beta}^{\bar{\alpha}_{\bar{\beta}}} \quad \text { and } \quad S_{\alpha \beta, \bar{\beta}}^{\alpha}-S_{\bar{\alpha} \beta, \beta}^{\bar{\alpha}_{\bar{\beta}}}=0 .
$$

Therefore we obtain

$$
\int_{M} S_{\lambda \alpha}^{\lambda} S_{\mu \bar{\mu}}^{\bar{\mu}} d V_{g}-\int_{M} S_{\alpha \beta, \bar{\beta}}^{\alpha} d V_{g}=\frac{1}{2} \int_{M} \delta \theta d V_{g}=0
$$

(4) is obvious by (1) and (3).

(5) follows from (4).

3. The asymptotic formulas for the Laplacians. Let $M$ be an $m$-dimensional compact Riemannian manifold and $\Lambda^{r}(M)$ be the space of exterior $r$-forms defined on $M$. We denote by $d$ and $\delta$ the exterior differential operator and its formal adjoint operator with respect to the Riemannian metric, respectively. The real Laplacian $\Delta_{r}$ acting on $\Lambda^{r}(M)$ is defined by $\Delta_{r}=d \delta+\delta d$.

Let $\left\{\lambda_{1}^{r}, \lambda_{2}^{r}, \ldots\right\}$ be the spectrum of $\Delta_{r}$ and let

$$
\sum_{j=1}^{\infty} \exp \left(-t \lambda_{j}^{r}\right) \underset{t \rightarrow+0}{\sim}(4 \pi t)^{-m / 2} \sum_{k=0}^{\infty} a_{k}^{r} t^{k}
$$

be the asymptotic expansion. Then we have

Theorem 3.1 (M. Berger [2] AND V. K. Patodi [11]).

$$
\begin{gathered}
a_{0}^{0}=\int_{M} d V_{g}, \quad a_{1}^{0}=\frac{1}{6} \int_{M} \tau d V_{g}, \quad a_{2}^{0}=\frac{1}{360} \int_{M} 5 \tau^{2}-2|\rho|^{2}+2|R|^{2} d V_{g}, \\
a_{0}^{1}=m \int_{M} d V_{g}, \quad a_{1}^{1}=\frac{m-6}{6} \int_{M} \tau d V_{g}, \\
a_{2}^{1}=\frac{m}{360} \int_{M} 5 \tau^{2}-2|\rho|^{2}+2|R|^{2} d V_{g}-\frac{1}{12} \int_{M}|R|^{2}-6|\rho|^{2}+2 \tau^{2} d V_{g},
\end{gathered}
$$

where $\tau, \rho, R$ denote the scalar curvature, the Ricci tensor and the curvature tensor, respectively. 
The coefficients $a_{0}^{0}, a_{1}^{0}$, and $a_{2}^{0}$ were computed by M. Berger [2] and the coefficients $a_{0}^{1}, a_{1}^{1}$, and $a_{2}^{1}$ were computed by V. K. Patodi [11]. Some of the other coefficients $a_{k}^{r}$ are computed by V. K. Patodi [11] and T. Sakai [12], etc.

Let $M$ be an $n$-dimensional compact Hermitian manifold. For $0 \leqslant p, q \leqslant n$, let $\Lambda^{p, q}(M)$ be the space of complex-valued $(p+q)$-forms of type $(p, q)$. We denote by $\bar{\partial}$ the differential operator $\bar{\partial}: \Lambda^{p, q}(M) \rightarrow \Lambda^{p, q+1}(M)$ and by $\vartheta$ its formal adjoint operator with respect to the Hermitian metric. With these, we define the complex Laplacian to be

$$
\square_{p, q}=\bar{\partial} \vartheta+\vartheta \bar{\partial}
$$

(cf. Morrow and Kodaira [10]).

Let $\left\{\lambda_{1}^{p, q}, \lambda_{2}^{p, q}, \ldots\right\}$ be the spectrum of $\square_{p, q}$ and let

$$
\sum_{j=1}^{\infty} \exp \left(-t \lambda_{j}^{p, q}\right) \underset{t \rightarrow+0}{\sim}(4 \pi t)^{-n} \sum_{k=0}^{\infty} b_{k}^{p, q} t^{k}
$$

be the asymptotic expansion. Then we have

TheOREM 3.2 (P. Gilkey [5] AND H. DonNelly [3]).

$$
\begin{aligned}
b_{1}^{0,0} & =\left(2^{n-1} / 12\right)\left\{2 \tilde{K}-K_{1}+6 K_{2}\right\}=\left(2^{n-1} / 6\right)\left\{K+3 K_{2}\right\}, \\
b_{1}^{1,0} & =\left(2^{n-1} / 12\right)\left\{2(n-3) \tilde{K}-n K_{1}+6 n K_{2}\right\} \\
& =\left(2^{n-1} / 12\right)\left\{2(n-3) K-3 K_{1}+6 n K_{2}\right\}, \\
b_{1}^{0,1} & =\left(2^{n-1} / 12\right)\left\{2(n-3) \tilde{K}+(12-n) K_{1}+6(n-4) K_{2}\right\} \\
& =\left(2^{n-1} / 12\right)\left\{2(n-3) K+9 K_{1}+6(n-4) K_{2}\right\},
\end{aligned}
$$

where $K=\int_{M} \tau d V_{g}, \tilde{K}=\int_{M} \tilde{\tau} d V_{g}, K_{1}=\int_{M} S_{\beta \gamma}^{\alpha} S_{\bar{\beta} \bar{\gamma}}^{\bar{\alpha}} d V_{g}, K_{2}=\int_{M} S_{\lambda \alpha}^{\lambda} S_{\mu \bar{\alpha}}^{\bar{\mu}} d V_{g}$ and $\tau$, $\tilde{\tau}, S$ denote the scalar curvature with respect to the Riemannian connection, the scalar curvature and the torsion tensor with respect to the Hermitian connection, respectively.

P. Gilkey has computed $b_{1}^{0,0}, b_{1}^{1,0}$, and $b_{1}^{0,1}$ using the other invariants in [5]. $\mathrm{H}$. Donnelly has asserted that $b_{k}^{p, q}$ should be described by the invariants of the Hermitian connection and has calculated the relations between Gilkey's invariants and the Hermitian invariants.

Corollary 3.3. Let $M$ and $M^{\prime}$ be compact connected Hermitian manifolds. Assume that $\operatorname{Spec}\left(M, \square_{p, q}\right)=\operatorname{Spec}\left(M^{\prime}, \square_{p, q}\right)$ for $(p, q)=(0,0),(0,1)$, and $(1,0)$. Then:

(1) $M$ is Kaehler if and only if $M^{\prime}$ is Kaehler.

(2) $M$ is semi-Kaehler if and only if $M^{\prime}$ is semi-Kaehler.

(3) When $\operatorname{dim}_{\mathrm{C}} M \geqslant 3, M$ is l.c. $K$. if and only if $M^{\prime}$ is l.c. $K$.

Proof. By Theorem 3.2, we have

$$
\int_{M} S_{\beta \gamma}^{\alpha} S_{\bar{\beta} \bar{\gamma}}^{\bar{\alpha}} d V_{g}=\int_{M^{\prime}} S_{\beta \gamma}^{\prime \alpha} S_{\bar{\beta} \bar{\gamma}}^{\prime \bar{\alpha}} d V_{g^{\prime}}
$$


and

$$
\int_{M} S_{\lambda \alpha}^{\lambda} S_{\mu \bar{\alpha}}^{\bar{\mu}} d V_{g}=\int_{M^{\prime}} S_{\lambda \alpha}^{\prime \lambda} S_{\bar{\mu} \bar{\alpha}}^{\prime \bar{\mu}} d V_{g^{\prime}}
$$

which, together with Proposition 2.4, imply these results.

REMARK. Under the same condition as Corollary 3.3, P. Gilkey proved that $M$ is Kaehler if and only if $M^{\prime}$ is Kaehler in [5]. Corollary 3.3 shows that the same results hold in the case of semi-Kaehler manifolds and 1.c.K. manifolds.

Corollary 3.4. If $\operatorname{Spec}\left(M, \square_{1,0}\right) \cup \operatorname{Spec}\left(M, \square_{0,1}\right)=\operatorname{Spec}\left(M, \frac{1}{2} \Delta_{1}\right)$, then $M$ is Kaehler.

Proof. By Theorems 3.1 and 3.2, we have $K_{1}+2(n-2) K_{2}=0$. When $n=1, M$ is always Kaehler. When $n \geqslant 2$, since $K_{1} \geqslant 0$ and $(n-2) K_{2} \geqslant 0$, we have $K_{1}=0$. Therefore $M$ is Kaehler.

REMARK. T. Tsujishita [6] proved that if $\operatorname{Spec}\left(M, \square_{0,0}\right)=\operatorname{Spec}\left(M, \frac{1}{2} \Delta_{0}\right)$ and $\operatorname{Spec}\left(M, \square_{1,0}\right) \cup \operatorname{Spec}\left(M, \square_{0,1}\right)=\operatorname{Spec}\left(M, \frac{1}{2} \Delta_{1}\right)$, then $M$ is Kaehler. The author showed that if $\operatorname{Spec}\left(M, \square_{0,0}\right)=\operatorname{Spec}\left(M, \frac{1}{2} \Delta_{0}\right)$, then $M$ is semi-Kaehler [13].

4. $P_{0} K$-manifolds. Vaisman [15] has studied the geometric structure of the particular classes of l.c.K. manifolds, i.e., $P K$-manifolds and $P_{0} K$-manifolds. In this section we will study the geometric structure of $P_{0} K$-manifolds precisely.

Definition 4.1 [15]. An 1.c.K. manifold is called a $P K$-manifold if its Lee form is parallel with respect to the Riemannian connection. If, moreover, the local conformal Kaehler metrics have vanishing curvature, the manifold is called a $P_{0} K$-manifold.

The Hopf manifolds are the typical examples of $P_{0} K$-manifolds. We will construct other examples of $P K$-manifolds. Let $S$ be a Sasakian manifold with the Sasakian structure $(\phi, \xi, \eta, g)$, where $\phi$ is a tensor field of type $(1,1), \xi$ a vector field, $\eta$ a 1 -form and $g$ a Riemannian metric on $S$. We introduce an almost complex structure $J$ on $S \times \mathbf{R}$ in the following manner: $J(X+f d / d t)=\phi X+f \xi-\eta(X) d / d t$, where $X$ is a tangent vector field of $S, t$ the coordinate of $\mathbf{R}$ and $f$ a differentiable function on $S \times \mathbf{R}$. This almost complex structure is integrable, and $(S \times \mathbf{R}, J, \tilde{g})$ is a Hermitian manifold, where $\tilde{g}$ is the product metric. Moreover it is 1.c.K. with Lee form $\omega=-d t$. It is easily seen that the Lee form is parallel. Thus we obtain examples of $P K$-manifolds.

Conversely Vaisman proved the following theorem.

THEOREM 4.2 (VAISMAN [15]). (i) The universal covering space of a connected complete $P K$-manifold is a product space $\tilde{S} \times \mathbf{R}$ of a simply connected complete Sasakian manifold $\tilde{S}$ and the real line $\mathbf{R}$.

(ii) The universal covering space of a $P_{0} K$-manifold is $\mathbf{C}^{n}-\{0\}$ with metric

$$
g=\left(c^{2} / \sum_{k=1}^{n} z_{k} \bar{z}_{k}\right) \sum_{j=1}^{n} d z_{j} d \bar{z}_{j},
$$

where $c$ is a positive constant.

Let $M$ be a connected complete $P K$-manifold with Lee form $\omega$ and $\tilde{M}$ be the universal covering space of $M$. We denote by $\pi$ the projection map $\pi: \tilde{M} \rightarrow M$. Then 
$\tilde{M}$ is also l.c.K. and its Lee form $\tilde{\omega}$ is given by $\tilde{\omega}=\pi * \omega$. By Theorem 4.2 , we have $\tilde{M}=\tilde{S} \times \mathbf{R}$ and $\tilde{\omega}=-d t$.

LEMMA 4.3. Let $\tilde{\Gamma}$ be the deck transformation group of the covering $\pi: \tilde{M} \rightarrow M$. Then any $\Phi \in \tilde{\Gamma}$ is given by $\Phi(x, t)=(\varphi(x), t+b)$ for $(x, t) \in \tilde{S} \times \mathbf{R}$, where $\varphi$ is an automorphism of the Sasakian manifold $\tilde{\boldsymbol{S}}$ and $b \in \mathbf{R}$.

Proof. $\Phi$ is a biholomorphic isometry on the 1.c.K. manifold $\tilde{M}$ and hence we have $\Phi^{*} \tilde{\omega}=\tilde{\omega}$. Since $\tilde{\omega}=-d t$, we get $\Phi^{*}(-d t)=-d t$ and $d\left(\Phi^{*} t-t\right)=0$. Therefore we have $\Phi^{*} t=t+b$. As $\Phi$ is a biholomorphic isometry, $\Phi$ transforms $\tilde{S} \times\{t\}$ to $\tilde{S} \times\{t+b\}$ preserving the Sasakian structure. From this we easily see that Lemma 4.3 holds.

By Lemma 4.3 we can introduce naturally a homomorphism of $\tilde{\Gamma}$ to $\mathbf{R}$, which we denote by $\sigma$. Namely, we define $\sigma$ by $\sigma(\Phi)=b$ if $\Phi^{*} t=t+b$. Ker $\sigma$ is a group acting on $\tilde{S} \times \mathbf{R}$ freely and properly discontinuously. We especially regard Ker $\sigma$ as a group acting on $\tilde{S}$ freely and properly discontinuously. Therefore $\tilde{S} \times$ $\mathbf{R} / \operatorname{Ker} \sigma=S \times \mathbf{R}$, where $S$ is a Sasakian manifold given by $\tilde{S} / \operatorname{Ker} \sigma$. We set $\Gamma=\tilde{\Gamma} / \operatorname{Ker} \sigma$. Regarding $\Gamma$ as the deck transformation group of the covering $S \times \mathbf{R} \rightarrow M$, we have $M=S \times \mathbf{R} / \Gamma$.

LemMa 4.4. If $S$ is compact, then $\Gamma$ is an infinite cyclic group generated by one generator.

PRoof. $\Gamma$ is isomorphic to the subgroup $\sigma(\Gamma)$ of $\mathbf{R}$. We show that $\sigma(\Gamma)$ is a discrete subgroup of $\mathbf{R}$. Unless $\sigma(\Gamma)$ is discrete, there exists a family of deck transformations $\left\{\Phi_{n}\right\}$ such that $\lim _{n \rightarrow \infty} \sigma\left(\Phi_{n}\right)=0$. By Lemma 4.3, $\Phi_{n}$ is given by $\Phi_{n}(x, t)=\left(\varphi_{n}(x), t+\sigma\left(\Phi_{n}\right)\right)$, where $\varphi_{n}$ is an automorphism of the Sasakian manifold $S$. For each $x \in S$, there exists a convergent subsequence $\varphi_{n_{k}}(x)$ such that $\lim _{k \rightarrow \infty} \varphi_{n_{k}}(x)=y$ for some point $y \in S$, because $S$ is compact. Therefore we have $\lim _{k \rightarrow \infty} \Phi_{n_{k}}(x, 0)=\lim _{k \rightarrow \infty}\left(\varphi_{n_{k}}(x), \sigma\left(\Phi_{n_{k}}\right)\right)=(y, 0)$, which is a contradiction to the fact that $\Gamma$ is a deck transformation group. Thus $\sigma(\Gamma)$ is a discrete subgroup of $\mathbf{R}$ and hence $\sigma(\Gamma)$ is an infinite cyclic group generated by one generator and so is $\Gamma$.

Especially by Lemma 4.4 we have

Proposition 4.5. Let $M$ be a compact connected $P_{0} K$-manifold with the Lee form of the constant length 1 . Then $M$ is $S \times \mathbf{R} / \Gamma$, where $S$ is a compact Sasakian manifold of constant sectional curvature 1 and $\Gamma$ is an infinite cyclic group generated by $\Phi$. Here $\Phi$ is given for $(x, t) \in S \times \mathbf{R}$ by $\Phi(x, t)=(\varphi(x), t+b)$, where $\varphi$ is an automorphism of the Sasakian manifold $S$ and $b$ is a nonzero real number.

We will consider the case when $S$ is $S^{2 n-1}(1)$. Let $W$ be $\mathbf{C}^{n}-\{0\}$ with metric $\|z\|^{-2} \sum_{j=1}^{n} d z_{j} d \bar{z}_{j}$. We denote by $g_{\mathbf{a}, b}$ the following biholomorphic isometry on $W$ :

$$
g_{\mathbf{a}, b}=e^{-2 \pi b}\left(\begin{array}{llll}
e^{2 \pi i a_{1}} & & & \\
& e^{2 \pi i a_{2}} & & \\
& & \ddots & \\
& & & e^{2 \pi i a_{n}}
\end{array}\right)
$$


where $b>0$ and $\mathbf{a}=\left(a_{1}, a_{2}, \ldots, a_{n}\right),-\frac{1}{2} \leqslant a_{1} \leqslant a_{2} \leqslant \ldots \leqslant a_{n}<\frac{1}{2}$. We denote by $G_{\mathbf{a}, b}$ the infinite cyclic group generated by $g_{\mathbf{a}, b}$. Obviously $G_{\mathbf{a}, b}$ acts on $W$ freely and properly discontinuously. Thus $M_{\mathbf{a}, b}=W / G_{\mathbf{a}, b}$ is an $n$-dimensional Hermitian manifold and, moreover, it is a $P_{0} K$-manifold. We note that when $a_{1}=a_{2}$ $=\cdots=a_{n}=a, M_{\mathrm{a}, b}$ is a Hopf manifold $M_{\alpha}$ with $\alpha=e^{-2 \pi(b-i a)}$.

The automorphism group preserving the Sasakian structure of $S^{2 n-1}(1)$ coincides with the unitary transformation group $U(n)$ of $\mathbf{C}^{n}$ restricted to $S^{2 n-1}(1)$. For any unitary transformation $A \in U(n)$, there exists $U \in U(n)$ such that

$$
U^{-1} A U=\left(\begin{array}{llll}
e^{2 \pi i a_{1}} & & & \\
& e^{2 \pi i a_{2}} & & \\
& & \ddots & \\
& & & e^{2 \pi i a_{n}}
\end{array}\right) .
$$

By these facts and Proposition 4.5, we have

Proposition 4.6. If $M$ satisfies the assumptions of Proposition 4.5 and, moreover, $S=S^{2 n-1}(1)$, then $M$ is biholomorphically isometric to an $M_{\mathrm{a}, b}$.

5. Eigenvalues on $M_{\mathrm{a}, b}$. In this section we will show

Proposition 5.1. Let $M_{\alpha}$ be a Hopf manifold with $\alpha=e^{-2 \pi(b-i a)}$. Assume that $b>1 / 2 \sqrt{2 n+1}$. If $\operatorname{Spec}\left(M_{\mathbf{a}^{\prime}, b^{\prime}}, \Delta_{0}\right)=\operatorname{Spec}\left(M_{\alpha}, \Delta_{0}\right)$ and $\operatorname{Spec}\left(M_{\mathbf{a}^{\prime}, b^{\prime}}, \square_{0,0}\right)$ $=\operatorname{Spec}\left(M_{\alpha}, \square_{0,0}\right)$, then $M_{\mathbf{a}^{\prime}, b^{\prime}}$ is bi- (or anti-) holomorphically isometric to the Hopf manifold $M_{\alpha}$.

We will prove the above result by comparing the eigenvalues of $M_{\alpha}$ and $M_{\mathrm{a}^{\prime}, b^{\prime}}$. As preliminaries, we review the results obtained by Bedford and Suwa [1]. Let $\Delta$ be the standard Laplacian on $\mathbf{C}^{n}$. Let $H_{p, q}$ be the space of harmonic polynomials of type $(p, q)$, i.e., the polynomials $f$ on $\mathbf{C}^{n}$ such that $\Delta f=0$ and $f(z)=\sum_{|\mu|=p .|\nu|=q} C_{\mu \nu} z^{\mu} \bar{z}^{\nu}$, where $\mu=\left(\mu_{1}, \mu_{2}, \ldots, \mu_{n}\right)$ and $\nu=\left(\nu_{1}, \nu_{2}, \ldots, \nu_{n}\right)$ are $n$-tuples of nonnegative integers, $|\mu|=\sum_{j=1}^{n} \mu_{j},|\nu|=\sum_{j=1}^{n} \nu_{j}$ and $z^{\mu}=z_{1}^{\mu_{1}} z_{2}^{\mu_{2}} \cdots z_{n}^{\mu_{n}}, \bar{z}^{\nu}=\bar{z}_{1}^{\nu} \bar{z}_{2}^{\nu_{2}} \cdots \bar{z}_{n}^{\nu_{n}}$.

LEMMA 5.2 (BEDFORD AND SUWA [1]). For $f \in H_{p, q}$ and for a complex number $\gamma$, on $W$,

$$
\square_{0,0}\left(\|z\|^{\gamma} f\right)=\left(-\frac{1}{2} \gamma^{2}-(p+q) \gamma+2 q(n-1)\right)\|z\|^{\gamma} f,
$$

and

$$
\Delta_{0}\left(\|z\|^{\gamma} f\right)=\left(-\gamma^{2}+2(p+q)(n-1-\gamma)\right)\|z\|^{\gamma} f .
$$

THEOREM 5.3 (BEDFORD AND SUWA [1]). (i) The eigenvalues of $\square_{0,0}$ on $M_{\alpha}$ are

$$
\frac{1}{2}(p+q)^{2}+\left(1 / 2 b^{2}\right)(a(p-q)-k)^{2}+2 q(n-1), \quad p, q \in \mathbf{Z}^{+}, \quad k \in \mathbf{Z} .
$$

(ii) The eigenvalues of $\Delta_{0}$ on $M_{\alpha}$ are

$$
(p+q)(p+q+2 n-2)+\left(1 / b^{2}\right)(a(p-q)-k)^{2}, \quad p, q \in \mathbf{Z}^{+}, \quad k \in \mathbf{Z},
$$

where $\mathbf{Z}^{+}$denotes the set of nonnegative integers and $\mathbf{Z}$ denotes the set of integers.

Lemma 5.4. Let $M_{\alpha}$ be a Hopf manifold which satisfies the assumption of Proposition 5.1. If $\operatorname{Spec}\left(M_{\mathbf{a}^{\prime}, b^{\prime}}, \Delta_{0}\right)=\operatorname{Spec}\left(M_{\alpha}, \Delta_{0}\right), b^{\prime}=b$ and $\left(a_{j}^{\prime}\right)^{2}=a^{2}$ for any $j$. 
Proof. If $\operatorname{Spec}\left(M_{\mathbf{a}^{\prime}, b^{\prime}}, \Delta_{0}\right)=\operatorname{Spec}\left(M_{\alpha}, \Delta_{0}\right)$, then the volumes of $M_{\mathbf{a}^{\prime}, b^{\prime}}$ and $M_{\alpha}$ are the same (cf. Theorem 3.1). Since $\operatorname{Vol}\left(M_{\mathrm{a}^{\prime}, b^{\prime}}\right)=2 \pi b^{\prime} \operatorname{Vol}\left(S^{2 n-1}(1)\right)$ and $\operatorname{Vol}\left(M_{\alpha}\right)$ $=2 \pi b \operatorname{Vol}\left(S^{2 n-1}(1)\right)$, we have $b^{\prime}=b$. By Theorem 5.3, it is easily seen that when $b>1 / 2 \sqrt{2 n+1}, a^{2} / b^{2}+2 n-1$ (with multiplicity $2 n$ ) and $k^{2} / b^{2}$ for some $k \in \mathbf{Z}$ are the only eigenvalues of $\Delta_{0}$ on $M_{\alpha}$ that are contained in the open interval $(0,4 n)$. Here we will show that $\left(a_{j}^{\prime}\right)^{2} / b^{\prime 2}+2 n-1 \in \operatorname{Spec}\left(M_{\mathbf{a}^{\prime}, b^{\prime}}, \Delta_{0}\right)$ for $j=1, \ldots, n$ and $k^{2} / b^{\prime 2} \in \operatorname{Spec}\left(M_{\mathbf{a}^{\prime}, b^{\prime}}, \Delta_{0}\right)$ for $k \in \mathbf{Z}$. If $\gamma=-1+i a_{j}^{\prime} / b^{\prime}$, then $\|z\|_{z_{j}}$ is $G_{\mathbf{a}^{\prime}, b^{\prime}}$ invariant. Therefore, by Lemma 5.2, $\|z\|^{\gamma} z_{j}$ is an eigenfunction of $\Delta_{0}$ on $M_{\mathbf{a}^{\prime}, b^{\prime}}$ with eigenvalue $\left(a_{j}^{\prime}\right)^{2} / b^{\prime 2}+2 n-1$. Similarly, for $\gamma=-1-i a_{j}^{\prime} / b^{\prime},\|z\|^{\gamma} \bar{z}_{j}$ is an eigenfunction with eigenvalue $\left(a_{j}^{\prime}\right)^{2} / b^{\prime 2}+2 n-1$. Moreover, when $\gamma=-i k / b^{\prime}$ for $k \in \mathbf{Z}$, $\|z\|^{\gamma}$ is $G_{\mathbf{a}^{\prime}, b^{\prime}}$-invariant and is an eigenfunction of $\Delta_{0}$ on $M_{\mathbf{a}^{\prime}, b^{\prime}}$ with eigenvalue $k^{2} / b^{\prime 2}$. Since $b^{\prime}=b>1 / 2 \sqrt{2 n+1}$, we have $0<\left(a_{j}^{\prime}\right)^{2} / b^{\prime 2}+2 n-1<4 n$. Comparing the eigenvalues which are contained in the open interval $(0,4 n)$, we obtain $\left(a_{j}^{\prime}\right)^{2}=a^{2}$ for all $j$.

RemarK. Let $M_{\alpha}$ be a Hopf manifold with $\alpha=e^{-2 \pi(b-i a)}$. If we put $a_{j}=-|a|$ for $j=1, \ldots, l$ and $a_{j}=|a|$ for $j=l+1, \ldots, n$, then $M_{\mathbf{a}, b}$ is isometric to $M_{\alpha}$ as a Riemannian manifold and hence $\operatorname{Spec}\left(M_{\mathrm{a}, b}, \Delta_{0}\right)=\operatorname{Spec}\left(M_{\alpha}, \Delta_{0}\right)$. But, in general, $M_{\mathrm{a}, b}$ is not biholomorphic to $M_{\alpha}$.

LEMMA 5.5. For a Hopf manifold $M_{\alpha}$, let $M_{\mathrm{a}, b}$ be the manifold defined in the remark. Assume, moreover, that $1 \leqslant l \leqslant n-1$ and $a \neq 0,-\frac{1}{2}$. Then

$$
\operatorname{Spec}\left(M_{\mathbf{a}, b}, \square_{0,0}\right) \neq \operatorname{Spec}\left(M_{\alpha}, \square_{0,0}\right) \text {. }
$$

Proof. We consider the case when $\mathbf{a}=(-a, \ldots,-a, a, \ldots, a)$. The case $\mathbf{a}=$ $(a, \ldots, a,-a, \ldots,-a)$ is similar. First we will show that $k^{2} / 2 b^{2}, \frac{1}{2}+(a+k)^{2} / 2 b^{2}$, and $2+k^{2} / 2 b^{2} \in \operatorname{Spec}\left(M_{\mathbf{a}, b}, \square_{0,0}\right)$ for $k \in \mathbf{Z}$. By the same argument as in the proof of Lemma 5.4 we have on $M_{\mathrm{a}, b}$ the following:

\begin{tabular}{c|c|c} 
functions & $\gamma$ & eigenvalues of $\square_{0,0}$ \\
\hline$\|z\|^{\gamma}$ & $-i k / b$ & $k^{2} / 2 b^{2}$ \\
\hline $\begin{array}{c}\|z\|^{\gamma} z_{j} \\
\text { for } 1 \leqslant j \leqslant l\end{array}$ & $-1-i(a+k) / b$ & $\frac{1}{2}+(a+k)^{2} / 2 b^{2}$ \\
\hline $\begin{array}{c}\|z\|^{\gamma}{ }^{2} \\
\text { for } l+1 \leqslant j \leqslant n\end{array}$ & $-1-i(a-k) / b$ & $1 / 2+(a-k)^{2} / 2 b^{2}$ \\
\hline $\begin{array}{c}\|z\|^{\gamma}{ }^{2} z_{i} \\
\text { for } 1 \leqslant i \leqslant l \\
\text { and } l+1 \leqslant j \leqslant n\end{array}$ & $-2+i k / b$ & $2+k^{2} / 2 b^{2}$
\end{tabular}

By Theorem 5.3(i) and the above results, we see that $\operatorname{Spec}\left(M_{\alpha}, \square_{0,0}\right)$ and $\operatorname{Spec}\left(M_{\mathrm{a}, b}, \square_{0,0}\right)$ have in common the eigenvalues $k^{2} / 2 b^{2}$ and $1 / 2+(a+k)^{2} / 2 b^{2}$ for $k \in \mathbf{Z}$. We remove these eigenvalues from $\operatorname{Spec}\left(M_{\alpha}, \square_{0,0}\right)$ and $\operatorname{Spec}\left(M_{\mathbf{a}, b}, \square_{0,0}\right)$, respectively, and denote the remainders by $\operatorname{Spec}\left(M_{\alpha}, \square_{0,0}\right)$ and $\operatorname{Spec}\left(M_{\mathrm{a}, b}, \square_{0,0}\right)$, respectively. We will show that if $\lambda \in \operatorname{Spec}\left(M_{\alpha}, \square_{0,0}\right)$, then $\lambda>2$. When $p+q \geqslant 3$, $(p+q)^{2} / 2+(a(p-q)-k)^{2} / 2 b^{2}+2 q(n-1) \geqslant \frac{q}{2}>2$ and when $q \geqslant 1$ the corresponding eigenvalues are greater than $2(n-1)$ and hence greater than 2 . For 
$p=2$ and $q=0$, the corresponding eigenvalues are $2+(2 a-k)^{2} / 2 b^{2}$. Since $a \neq 0,-\frac{1}{2}$, these eigenvalues are actually greater than 2 . The eigenvalues for $p=1$ and $q=0$ or $p=0$ and $q=0$ are already removed. Thus we have $\lambda>2$ for any $\lambda \in \operatorname{Spec}\left(M_{\alpha}, \square_{0,0}\right)$. However $\operatorname{Spec}\left(M_{\mathrm{a}, b}, \square_{0,0}\right)$ contains 2. In fact when $\gamma=-2$, $\|z\|^{\gamma}{ }_{z_{i}} z_{j}$ is an eigenfunction of $\square_{0,0}$ on $M_{\mathrm{a}, b}$ with eigenvalue 2 for $1 \leqslant i \leqslant l$ and $l+1 \leqslant j \leqslant n$. Therefore we obtain $\operatorname{Spec}\left(M_{\mathrm{a}, b}, \square_{0,0}\right) \neq \operatorname{Spec}\left(M_{\alpha}, \square_{0,0}\right)$.

Proof of Proposition 5.1. By Lemmas 5.4 and 5.5 we see that $M_{\mathbf{a}^{\prime}, b^{\prime}}$ is biholomorphically isometric to $M_{\alpha}$ or to $M_{\bar{\alpha}}$, where $\bar{\alpha}$ is a complex conjugate of $\alpha$.

REMARK. Since

$$
\operatorname{Spec}\left(M_{\alpha}, \Delta_{0}\right)=\operatorname{Spec}\left(M_{\bar{\alpha}}, \Delta_{0}\right)
$$

and

$$
\operatorname{Spec}\left(M_{\alpha}, \square_{0,0}\right)=\operatorname{Spec}\left(M_{\bar{\alpha}}, \square_{0,0}\right),
$$

we cannot distinguish between $M_{\alpha}$ and $M_{\bar{\alpha}}$ by the spectrum of $\Delta_{0}$ and $\square_{0,0}$.

6. Proof of Theorem. Using the asymptotic invariants we will prove first that $M$ is also a $P_{0} K$-manifold. Secondly, comparing the eigenvalues, we obtain the result.

Proposition 6.1. Let $M$ and $M^{\prime}$ be compact connected Riemannian manifolds with $\operatorname{dim}_{\mathbf{R}} M=m(\geqslant 4)$. Assume that $M^{\prime}$ is conformally flat and has a constant scalar curvature $\tau^{\prime}$. If $\operatorname{Spec}\left(M, \Delta_{0}\right)=\operatorname{Spec}\left(M^{\prime}, \Delta_{0}\right)$ and $\operatorname{Spec}\left(M, \Delta_{1}\right)=\operatorname{Spec}\left(M^{\prime}, \Delta_{1}\right)$, then $M$ is also conformally flat and has the same constant scalar curvature $\tau$. Moreover $\int_{M}|\rho|^{2} d V_{g}=\int_{M^{\prime}}\left|\rho^{\prime}\right|^{2} d V_{g^{\prime}}$.

Proof. Let $C$ and $C^{\prime}$ be the Weyl conformal curvature tensors of $M$ and $M^{\prime}$, respectively. Since $a_{2}^{0}=a_{2}^{\prime 0}$ and $a_{2}^{1}=a_{2}^{\prime 1}$, using the formula $|C|^{2}=|R|^{2}-$ $4|\rho|^{2} /(m-2)+2 \tau^{2} /(m-1)(m-2)$ and Theorem 3.1 we have

$$
\int_{M}\left\{5(m-2)|C|^{2}+\frac{13 m^{3}-67 m^{2}+100 m-36}{(m-1)(m-2)} \tau^{2}\right\} d V_{g}
$$

$$
=\int_{M^{\prime}}\left\{5(m-2)\left|C^{\prime}\right|^{2}+\frac{13 m^{3}-67 m^{2}+100 m-36}{(m-1)(m-2)} \tau^{\prime 2}\right\} d V_{g^{\prime}} \text {. }
$$

We note that $13 m^{3}-67 m^{2}+100 m-36>0$ when $m \geqslant 3$. Since $\tau^{\prime}$ is constant, $a_{0}^{0}=a_{0}^{\prime 0}$ and $a_{1}^{0}=a_{1}^{\prime 0}$ imply that $\int_{M} \tau^{2} d V_{g} \geqslant \int_{M^{\prime}} \tau^{\prime 2} d V_{g^{\prime}}$. In fact, using Schwarz's inequality, we have

$$
\begin{aligned}
\int_{M} d V_{g} \int_{M} \tau^{2} d V_{g} & \geqslant\left(\int_{M} \tau d V_{g}\right)^{2}=\left(\int_{M^{\prime}} \tau^{\prime} d V_{g^{\prime}}\right)^{2}=\tau^{\prime 2}\left(\int_{M^{\prime}} d V_{g^{\prime}}\right)^{2} \\
& =\int_{M^{\prime}} d V_{g^{\prime}} \int_{M^{\prime}} \tau^{\prime 2} d V_{g^{\prime}}=\int_{M} d V_{g} \int_{M^{\prime}} \tau^{\prime 2} d V_{g^{\prime}}
\end{aligned}
$$

Moreover since $M^{\prime}$ is conformally flat (i.e., $C^{\prime}=0$ ), it follows from (*) that $M$ is conformally flat and has constant scalar curvature $\tau=\tau^{\prime}$. From these results and $a_{2}^{0}=a_{2}^{\prime 0}$, we obtain $\int_{M}|\rho|^{2} d V_{g}=\int_{M^{\prime}}\left|\rho^{\prime}\right|^{2} d V_{g^{\prime}}$. 
LEMMA 6.2. Let $(M, g)$ be an $n$-dimensional $(n \geqslant 3)$ conformally flat l.c. K. manifold with Lee form $\omega$. Then

$$
\begin{gathered}
\rho_{j i}=2(n-1)\left(-\nabla_{j} \omega_{i}-\omega_{j} \omega_{i}+|\omega|^{2} g_{j i}\right)+\delta \omega g_{j i}, \\
\tau=2(2 n-1) \delta \omega+2(n-1)(2 n-1)|\omega|^{2}, \\
|\rho|^{2}=4(n-1)^{2}|\nabla \omega|^{2}+8(n-1)^{2} \nabla_{j} \omega_{i} \omega^{j} \omega^{i}+4(n-1)(4 n-3)|\omega|^{2} \delta \omega \\
+4(n-1)^{2}(2 n-1)|\omega|^{4}+2(3 n-2)(\delta \omega)^{2},
\end{gathered}
$$

where $\nabla$ denotes covariant differentiation with respect to the Riemannian connection of $(M, g)$. Moreover if $M$ is compact, then

$$
\begin{gathered}
\int_{M} \nabla_{j} \omega_{i} \omega^{j} \omega^{i} d V_{g}=\frac{1}{2} \int_{M}|\omega|^{2} \delta \omega d V_{g} \\
\int_{M}|\nabla \omega|^{2} d V_{g}=(n-2) \int_{M}|\omega|^{2} \delta \omega d V_{g}+\int_{M}(\delta \omega)^{2} d V_{g} .
\end{gathered}
$$

Proof. By the usual calculations of the conformal changes of Riemannian metrics, we get (1), (2), and (3). Integrating the equation

$$
\delta\left(|\omega|^{2} \omega\right)=-2 \nabla_{j} \omega_{i} \omega^{j} \omega^{i}+|\omega|^{2} \delta \omega
$$

on $M$ we obtain (4). The integral formula

$$
\int_{M}\left\{|\nabla \omega|^{2}-(\delta \omega)^{2}-\frac{1}{2}|d \omega|^{2}+\rho_{j i} \omega^{j} \omega^{i}\right\} d V_{g}=0
$$

together with (1) and (4) implies (5).

Proposition 6.3. Under the assumptions of Theorem, $M$ is also a $P_{0} K$-manifold and the length of its Lee form $\omega$ is 1.

Proof. We note that the Hopf manifold $M_{\alpha}$ is a $P_{0} K$-manifold and the length of its Lee form is 1 . Moreover $M_{\alpha}$ has constant scalar curvature $\tau^{\prime}=2(n-1)(2 n-1)$. By Corollary 3.3(3), $M$ is also 1.c.K.. We denote its Lee form by $\omega$. By Proposition 6.1 $M$ is a conformally flat manifold with constant scalar curvature $\tau=$ $2(n-1)(2 n-1)$. Let $\rho$ and $\rho^{\prime}$ be the Ricci tensors of $M$ and $M_{\alpha}$, respectively. Then Proposition 6.1 implies that $\int_{M}|\rho|^{2} d V_{g}=\int_{M_{\alpha}}\left|\rho^{\prime}\right|^{2} d V_{g^{\prime}}$. Using Lemma 6.2(2), (3), (4), and (5) and the fact that the scalar curvature $\tau$ of $M$ is constant, we obtain

$$
\int_{M}|\rho|^{2} d V_{g}=-2(n-2) \int_{M}(\delta \omega)^{2} d V_{g}+\frac{1}{2 n-1} \int_{M} \tau^{2} d V_{g}
$$

On the other hand,

$$
\int_{M_{\alpha}}\left|\rho^{\prime}\right|^{2} d V_{g^{\prime}}=\frac{1}{2 n-1} \int_{M_{\alpha}} \tau^{\prime 2} d V_{g^{\prime}}
$$

Therefore we have $\delta \omega=0$ on $M$. From this it follows from Lemma 6.2(5) that $\nabla \omega=0$. By Lemma 6.2(2), we have $|\omega|=1$. 
LEMMA 6.4. Under the assumptions of Theorem, $M$ is biholomorphically isometric to an $M_{\mathbf{a}, b}$.

Proof. From Propositions 4.5 and 6.3 it follows that $M$ is $S \times \mathbf{R} / \Gamma$, where $S$ is a compact Sasakian manifold of constant sectional curvature 1 and $\Gamma$ is an infinite cyclic group generated by $\Phi$ which is given by $\Phi(x, t)=\left(\varphi(x), t+2 \pi b^{\prime}\right)$ for $(x, t) \in S \times \mathbf{R}$. Let $S^{1}$ be $\mathbf{R} / 2 \pi b^{\prime} \mathbf{Z}$ and $p^{\prime}$ the covering $p^{\prime}: \mathbf{R} \rightarrow S^{1}$. Let $p^{\prime \prime}$ be the natural projection $p^{\prime \prime}: S \times \mathbf{R} \rightarrow \mathbf{R}$ and $\pi=p^{\prime} \circ p^{\prime \prime}: S \times \mathbf{R} \rightarrow S^{1}$. Since $\pi \circ \Phi=\pi$, we can define the mapping $\pi: M \rightarrow S^{1}$. From the constructions we see that $\pi$ is a Riemannian submersion with totally geodesic fibres $S$. Let $\Delta_{S}$ be the Laplacian acting on $\Lambda^{0}(S)$. We naturally regard $\Delta_{S}$ as a differential operator acting on $\Lambda^{0}(S \times \mathbf{R})$. Since $\Phi^{*} \Delta_{S}=\Delta_{S}$, we can regard $\Delta_{S}$ as a differential operator acting on $\Lambda^{0}(M)$. From the constructions we see that $\Delta_{0} \Delta_{S}=\Delta_{S} \Delta_{0}$, where $\Delta_{0}$ is the real Laplacian of $M$ acting on $\Lambda^{0}(M)$. Moreover we have $\left\langle\Delta_{S} f_{1}, f_{2}\right\rangle=\left\langle f_{1}, \Delta_{S} f_{2}\right\rangle$ for $f_{1}$ and $f_{2} \in \Lambda^{0}(M)$, where $\langle$,$\rangle denotes the scalar product in \Lambda^{0}(M)$. Let $V_{\lambda}$ be an eigenspace of $\Delta_{0}$ on $M$ corresponding to the eigenvalue $\lambda$. Then $V_{\lambda}$ is decomposed as $V_{\lambda}=V\left(\mu_{k}\right)+\cdots+V\left(\mu_{0}\right)$, where $\Delta_{s} f=\mu_{j} f$ for $f \in V\left(\mu_{j}\right)$ and $\mu_{k}>\mu_{k-1}$ $>\cdots>\mu_{0}=0$. We easily see that $\lambda \geqslant \mu_{j}$ for $j=0,1, \ldots, k$ and $\mu_{j}$ is an eigenvalue of $\Delta_{S}$ acting on $\Lambda^{0}(S)$. If $V_{\lambda}=V\left(\mu_{0}\right)$, for $f \in V_{\lambda}$ there exists an eigenfunction $f^{*}$ of $-d^{2} / d t^{2}$ on $S^{1}$ such that $\pi^{*} f^{*}=f$ and, in particular, we have $\lambda=k^{2} / b^{\prime 2}$ for $k \in \mathbf{Z}$. Under the above preliminaries, we will show that $S$ is isomorphic to $S^{2 n-1}(1)$. If $S$ is not simply connected, we have $\mu_{j} \geqslant 4 n$ for $j \neq 0$. Therefore we have $\lambda \geqslant 4 n$ unless $V_{\lambda}=V\left(\mu_{0}\right)$. Hence $\operatorname{Spec}\left(M, \Delta_{0}\right)$ contains in the open interval $(0,4 n)$ only eigenvalues that are of the form $k^{2} / b^{\prime 2}$ for some $k \in \mathbf{Z}$. In particular, there exists no eigenvalue of $\Delta_{0}$ on $M$ with multiplicity $2 n$ in the interval $(0,4 n)$. On the other hand, if $b>1 / 2 \sqrt{2 n+1}$, then $\operatorname{Spec}\left(M_{\alpha}, \Delta_{0}\right)$ contains in $(0,4 n)$ the eigenvalue $a^{2} / b^{2}+$ $2 n-1$ with multiplicity $2 n$. This is incompatible with $\operatorname{Spec}\left(M_{\alpha}, \Delta_{0}\right)=\operatorname{Spec}\left(M, \Delta_{0}\right)$. Therefore $S$ is simply connected. Hence it follows from Proposition 4.6 that $M$ is biholomorphically isometric to an $M_{\mathrm{a}, b}$.

Proof of Theorem. Lemma 6.4 and Proposition 5.1 bring the proof of Theorem to completion if we note the following fact.

LEMMA 6.5. Let $\bar{\alpha}$ be the complex conjugate of $\alpha$ and assume that $\alpha \neq \bar{\alpha}$. Then $\operatorname{Spec}\left(M_{\alpha}, \square_{0,1}\right) \neq \operatorname{Spec}\left(M_{\bar{\alpha}}, \square_{0,1}\right)$.

Proof. Suppose that $\operatorname{Spec}\left(M_{\alpha}, \square_{0,1}\right)=\operatorname{Spec}\left(M_{\bar{\alpha}}, \square_{0,1}\right)$. Since $\operatorname{Spec}\left(M_{\bar{\alpha}}, \square_{0,1}\right)$ $=\operatorname{Spec}\left(M_{\alpha}, \square_{1,0}\right)$, we have $\operatorname{Spec}\left(M_{\alpha}, \square_{0,1}\right)=\operatorname{Spec}\left(M_{\alpha}, \square_{1,0}\right)$. Hence the asymptotic invariants $b_{1}^{0,1}$ and $b_{1}^{1,0}$ coincide on $M_{\alpha}$. Therefore it follows that

$$
\int_{M_{\alpha}} S_{\beta \gamma}^{\alpha} S_{\bar{\beta} \bar{\gamma}}^{\bar{\alpha}} d V_{g}-2 \int_{M_{\alpha}} S_{\lambda \alpha}^{\lambda} S_{\mu \bar{\alpha}}^{\bar{\mu}} d V_{g}=0
$$

However on $M_{\alpha}$,

$$
\int_{M_{\alpha}} S_{\beta \gamma}^{\alpha} S_{\bar{\beta} \bar{\gamma}}^{\bar{\alpha}} d V_{g}-2 \int_{M_{\alpha}} S_{\lambda \alpha}^{\lambda} S_{\mu \bar{\alpha}}^{\bar{\mu}} d V_{g}=-4(n-1)(n-2) \operatorname{Vol}\left(M_{\alpha}\right),
$$

which is a contradiction. 


\section{REFERENCES}

1. E. Bedford and T. Suwa, Eigenvalues of Hopf manifolds, Proc. Amer. Math. Soc. 60 (1976), 259-264.

2. M. Berger, Le spectre des variétés riemanniennes, Rev. Roumaine Math. Pures Appl. 13 (1968), 915-931.

3. H. Donnelly, Invariance theory of Hermitian manifolds, Proc. Amer. Math. Soc. 58 (1976), 229-233.

4. P. Gauduchon, Fibrés Hermitiens à endomorphisme de Ricci non negatif, Bull. Soc. Math. France 105 (1977), 113-140.

5. P. Gilkey, Spectral geometry and the Kaehler condition for complex manifolds, Invent. Math. 26 (1974), 231-258.

6. Correction to spectral geometry and the Kaehler condition for complex manifolds, Invent. Math. 29 (1975), 81-82.

7. A Gray, Some examples of almost Hermitian manifolds, Illinois J. Math. 10 (1966), 353-366.

8. T. Kashiwada, Some properties of locally conformal Kaehler manifolds, Hokkaido Math. J. 8 (1979), 191-198.

9. S. Kobayashi and K. Nomizu, Foundations of differential geometry. II, Interscience, New York, 1969.

10. J. Morrow and K. Kodaira, Complex manifolds, Holt, Rinehart and Winston, New York, 1971.

11. V. Patodi, Curvature and the fundamental solution of the heat operator, J. Indian Math. Soc. 34 (1970), 269-285.

12. T. Sakai, On eigenvalues of Laplacian and curvature of Riemannian manifold, Tôhoku Math. J. 23 (1971), 589-603.

13. K. Tsukada, Eigenvalues of the Laplacian on Calabi-Eckmann manifolds, J. Math. Soc. Japan 33 (1981), 673-691.

14. I. Vaisman, On locally conformal almost Kaehler manifolds, Israel J. Math. 24 (1976), 338-351.

15. Locally conformal Kaehler manifolds with parallel Lee form, Rend. Mat. 12 (1979), 263-284.

16. , Remarkable operators and commutation formulas on locally conformal Kaehler manifolds, Compositio Math. 40 (1980), 287-299.

Department of Mathematics, Tokyo Metropolitan University, Setagaya, Tokyo, 158, Japan 